\title{
OLA1 is responsible for normal spindle assembly and SAC activation in mouse oocytes
}

\author{
Di Xie ${ }^{1,2}$, Juan Zhang ${ }^{2}$, JinLi Ding ${ }^{1}$, Jing Yang ${ }^{\text {Corresp., } 1}$, Yan Zhang ${ }^{\text {Corresp. } 3}$ \\ 1 Reproductive Medical Center, Renmin Hospital of Wuhan University, WuHan, HuBei, China \\ 2 Reproductive Medical Center, General Hospital of Central Theater Command, WuHan, HuBei, China \\ 3 Department of Clinical Laboratory, Renmin Hospital of Wuhan University, WuHan, HuBei, China \\ Corresponding Authors: Jing Yang, Yan Zhang \\ Email address: dryangqing@hotmail.com, peneyyan@mail.ustc.edu.cn
}

Background. OLA1 is a member of the GTPase protein family, unlike other members, it possess both GTPase and ATPase activities, and can bind and hydrolyze ATP more efficiently than GTP. OLA1 participates in cell proliferation, oxidative response, protein synthesis and tumorigenesis. However, whether OLA1 is also required for oocyte meiosis is still unknown.

Methods. In this study, the localization, expression, and functions of OLA1 in the mouse oocyte meiosis were examined. Immunofluorescent and confocal microscopy were used to explore the location pattern of OLA1 in the mouse oocyte. Moreover, nocodazole treatment was used to confirm the spindle-like location of OLA1 during mouse meiosis. Western blot was used to explore the expression pattern of OLA1 in the mouse oocyte. Microinjection of siRNA was used to explore the OLA1 functions in the mouse oocyte meiosis. In addition, chromosome spreading was used to investigate the spindle assembly checkpoint (SAC) activity.

Results. Immunofluorescent staining showed that OLAl evenly distributed in the cytoplasm at germinal vesicle (GV) stage. After meiosis resumption (GVBD), OLA1 co-localized with spindles, which was further identified by nocodazole treatment experiments. Knockdown of OLA1 impaired the germinal vesicle breakdown progression and finally resulted in a lower polar body extrusion rate. Immunofluorescence analysis indicated that knockdown of OLA1 led to abnormal spindle assembly, which was evidenced by multipolar spindles in OLA1-RNAi-oocytes. After $6 \mathrm{~h}$ post-GVBD in culture, an increased proportion of oocyte which has precociously entered into anaphase/telephase I (A/TI) was observed in OLA1knockdown oocytes, suggesting that loss of OLA1 resulted in the premature segregation of homologous chromosomes. In addition, the chromosome spread analysis suggested that OLA1 knockdown induced premature anaphase onset was due to the precocious inactivation of SAC. Taken together, we concluded that OLA1 plays important role in GVBD, spindle assembly and SAC activation maintenance in oocyte meiosis. 
1

3 Di Xie ${ }^{1,2}$, Juan Zhang ${ }^{2}$, Jinli Ding ${ }^{1}$, Jing Yang ${ }^{1, *}$ and Yan Zhang ${ }^{3, *}$

4

$5 \quad{ }^{1}$ Reproductive Medical Center, Renmin Hospital of Wuhan University, Wuhan /Hubei, PR

6 China;

$7 \quad{ }^{2}$ Reproductive Medical Center, General Hospital of Central Theater Command, Wuhan /Hubei,

8 PR China;

$9{ }^{3}$ Department of Clinical Laboratory, Renmin Hospital of Wuhan University, Wuhan /Hubei, PR

10 China;

11

12

13

14

15

16

\section{OLA1 is responsible for normal spindle assembly} and SAC activation in mouse oocytes

Corresponding Authors:

Jing Yang ${ }^{1}$

No.238 Jiefang Road, Wuchang District, Wuhan, Hubei Province, 430060, PR China Email address: dryangqing@hotmail.com

Yan Zhang ${ }^{3}$

No.238 Jiefang Road, Wuchang District, Wuhan, Hubei Province, 430060, PR China

Email address: peneyyan@mail.ustc.edu.cn 


\section{ABSTRACT}

34 Background. OLA1 is a member of the GTPase protein family, unlike other members, it possess both GTPase and ATPase activities, and can bind and hydrolyze ATP more efficiently than GTP.

OLA1 participates in cell proliferation, oxidative response, protein synthesis and tumorigenesis.

However, whether OLA1 is also required for oocyte meiosis is still unknown.

Methods. In this study, the localization, expression, and functions of OLA1 in the mouse oocyte meiosis were examined. Immunofluorescent and confocal microscopy were used to explore the location pattern of OLA1 in the mouse oocyte. Moreover, nocodazole treatment was used to confirm the spindle-like location of OLA1 during mouse meiosis. Western blot was used to explore the expression pattern of OLA1 in the mouse oocyte. Microinjection of siRNA was used to explore the OLA1 functions in the mouse oocyte meiosis. In addition, chromosome spreading was used to investigate the spindle assembly checkpoint (SAC) activity.

Results. Immunofluorescent staining showed that OLA1 evenly distributed in the cytoplasm at germinal vesicle (GV) stage. After meiosis resumption (GVBD), OLA1 co-localized with spindles, which was further identified by nocodazole treatment experiments. Knockdown of OLA1 impaired the germinal vesicle breakdown progression and finally resulted in a lower polar body extrusion rate. Immunofluorescence analysis indicated that knockdown of OLA1 led to abnormal spindle assembly, which was evidenced by multipolar spindles in OLA1-RNAi oocytes. After 6 h post-GVBD in culture, an increased proportion of oocyte which has precociously entered into anaphase/telephase I (A/TI) was observed in OLA1-knockdown oocytes, suggesting that loss of OLA1 resulted in the premature segregation of homologous chromosomes. In addition, the chromosome spread analysis suggested that OLA1 knockdown 
55 induced premature anaphase onset was due to the precocious inactivation of SAC. Taken

56 together, we concluded that OLA1 plays important role in GVBD, spindle assembly and SAC

57 activation maintenance in oocyte meiosis.

\section{INTRODUCTION}

59 Mammalian gametes are yieled through an event named meiosis where two consecutive divisions

60 are conducted to halve the chromosomes without an intervening replicative process (Verlhac et

61 al., 2016). Spindle assembly and chromosome segregation are both vital courses to keep genomic

62 stability during oocyte meiosis. Errors in these two processes can lead to the failure of meiosis or

63 the generation of aneuploidy (Ma et al., 2014; Jiao et al., 2017; Han et al., 2015). Moreover, human

64 pregnancy loss has strong correlation with fetal aneuploidy which can be induced by defective

65 spindle structure and abnormal chromosome segregation (Webster et al., 2017). To guarantee the

66 normal progression of oocyte meiosis, spindle assembly and chromosome segregation must be

67 correctly commanded.

68 After germinal vesicle breakdown (GVBD), as absent of centrosome at the mitosis, the

69 meiotic spindle is emanated and nucleated from microtubule organizing centers (MTOCs) (Schuh

70 et al., 2007; Clift et al., 2015; Bennabi et al., 2016). At this time, chromosomes and spindle

71 microtubules has begun to interact with each other. Chromosomes continuously move to the

72 equatorial plate as the cell cycle progresses, and the alignment of all chromosomes in this

73 process does not coincide at the same time. Spindle assembly checkpoint (SAC) signaling

74 controls this unevenly process to arrest oocyte at pre-metaphase I stage to avoid the premature

75 chromosome segregation (Vogt et al., 2008; Touati et al., 2016; Sanders et al., 2018). Once the

76 incorrect connection of chromosome with microtubule is established, all chromosomes are

77 correctly aligned at the metaphase I plate (MI stage) then SAC proteins will dissociate from 
78 kinetochore to trigger the segregation of chromosomes (anaphase onset), which finally bring out

79 the polar body extrusion and oocyte will arrest at metaphase II (MII).

80 OLA1 is a member of the GTPase protein family, unlike other members, it can bind and

81 hydrolyze both ATP and GTP (Koller-Eichhorn et al., 2007). OLA1 can act as a negative regulator 82 of the antioxidative response via nontranscriptional mechanisms (Zhang et al., 2009). Moreover,

83 OLA1 involves in eukaryotic initiation factor 2 (eIF2)-mediated protein synthesis (Chen et al.,

84 2015; Ding et al., 2016). During mitosis, OLA1 localizes to centrosomes in interphase and then to

85 the spindle pole after nuclear envelop breakdown. OLA1 can directly interact with BRCA1 and $86 \gamma$-tubulin by bounding to the amino-terminal of these two proteins, and this interaction is very 87 important for the centrosomal regulation (Matsuzawa et al., 2014). And knockdown of OLA1 88 results in the centrosome amplification of centrosome and the disorders in microtubule aster 89 formation (Matsuzawa et al., 2014; Yoshino et al., 2018). It is noteworthy that, BRCA1 has been 90 shown to exert functions in oocyte meiosis, and knockdown of BRCA1 in mouse oocyte causes 91 abnormal spindle assembly and the dysfunction of SAC (Xiong et al., 2008). As an interacting 92 factor, whether OLA1 also participates in meiotic progression remains elusive. Thus, we attempt 93 to explore the possible roles of OLA1 in oocyte meiosis.

94 Oocyte maturation is a complicated process, which can be affected by many factors including 95 functional cellular proteins, and whether OLA1 is involve in meiosis is totally unknown. In this 96 study, we have found that OLA1 participates in oocyte meiotic maturation, especially in the 97 progress of germinal vesicle breakdown, spindle assembly and SAC activation.

MATERIALS AND METHODS Animals

Three-four weeks-old female KM mice were used in this experiment. Animal experiments were approved by Hubei Research Center of Laboratory Animal (Approval ID: SYXK (Hubei) 2014- 
102 0082SCXK). Animal care and handling were conformed to regulations of Animal Care and Use

103 Committee of General hospital of the Central Theater Command.

104 Antibodies and chemicals

105 All chemicals and culture media were purchased from Sigma (St Louis, MO) unless those

106 specifically mentioned. Rabbit polyclonal anti-OLA1 antibody (Cat\# A4673) was purchased

107 from ABclonal (Wuhan, China). Mouse monoclonal anti- $\alpha$-tubulin-FITC antibody (Cat\# F2168)

108 was obtained from Sigma (St Louis, MO); Sheep polyclonal anti-BubR1 antibody (Cat\# 28193)

109 was obtained from Abcam (Cambridge, UK). FITC-conjugated donkey anti-sheep $\operatorname{IgG}(\mathrm{H}+\mathrm{L})$

110 was purchased from Jackson ImmunoResearch Laboratory.

\section{Oocyte collection and culture}

112 To harvest fully grown GV oocyte, 3-4 weeks-old female KM mice were firstly intraperitoneally

113 injected with 5 IU pregnant mares serum gonadotropin, after 46-48h, mice were sacrificed by

114 cervical dislocation and ovaries were isolated. Enclosed cumulus oocytes were removed by

115 repeatedly pipetting, and then oocytes were cultured in pre-warmed M16 medium under paraffin

116 oil at $37^{\circ} \mathrm{C}$ in a $5 \% \mathrm{CO} 2$ atmosphere. At appropriate time points, oocytes were selected for

117 different experiments. In order to inhibit the spontaneous meiotic resume in vitro culture, M2

118 medium with $50 \mu \mathrm{M}$ IBMX was used to maintain the GV stage.

119 Nocodazole treatment and recovery

120 To destroy the spindle apparatus, wild-type MI and MII stage oocytes were cultured in M16

121 medium with $20 \mu \mathrm{g} / \mathrm{ml}$ of nocodazole for $20 \mathrm{~min}$. To re-assemble spindle microtubule,

122 nocodazole was washed outand oocytes were cultured in fresh M16 medium for 30 min.

123 Microinjection of OLA1 siRNA

124 OLA1 siRNA (sc-145833; Santa Cruz, CA) was used to knockdown OLA1. Control siRNA (sc-

125 37007; Santa Cruz, CA) was used as negative control. For microinjection, $5 \mathrm{pL}$ of $30 \mu \mathrm{M}$ 
126 control siRNA and OLA1 siRNA were injected into fully grown GV oocytes, to completely

127 degrade thetargeted mRNA, oocytes were cultured in M2 medium with $50 \mu \mathrm{M}$ IBMX for $24 \mathrm{~h}$.

128 After that, oocytes were directly collected for western blotting or culture in fresh M16 medium

129 for meiotic maturation.

130 Immunofluorescence analysis

131 Oocytes were fixed and permeabilized in PBS containing 4\% paraformaldehyde, 0.5\% Triton X-

$132100(\mathrm{pH}=7.4)$ for $40-50 \mathrm{~min}$. Then, oocytes were blocked in PBS containing $2 \% \mathrm{BSA} 1 \mathrm{~h}$ at

133 room temperature and incubated overnight at $4^{\circ} \mathrm{C}$ with anti-OLA1 antibody (ABclonal, 1:100),

134 anti- $\alpha$-tubulin-FITC antibody (Sigma, 1:100). After washing 3 times in PBS containing $0.05 \%$

135 Tween-20, oocytes were incubated with appropriate secondary antibodies for $1 \mathrm{~h}$ at $37^{\circ} \mathrm{C}$.

136 Chromosomes were visualized by staining with $1 \mu \mathrm{g} / \mathrm{ml}$ of DAPI for 5-10 min at room

137 temperature. Finally, oocytes were mounted on slides and immunofluorescent images were

138 observed by a confocal laser scanning microscope (Zeiss LSM 800, Germany). Non-immunized

139 rabbit or mouse IgG was used as negative control.

140 Western blot

141 Oocytes were placed in $2 \mathrm{X}$ SDS loading buffer and boiled for $5 \mathrm{~min}$ at $95^{\circ} \mathrm{C}$. The proteins were

142 separated by SDS-PAGE and then electrophoretically transferred to polyvinylidene fluoride

143 (PVDF) membranes. After transfer, the PVDF membranes were blocked in TBST containing 5\%

144 non-fat milk for $1 \mathrm{~h}$, followed by incubation with OLA1 antibody (ABclonal, 1:1000) overnight

145 at $4^{\circ} \mathrm{C}$. After three times washes in TBST buffer, the membranes were incubated with HRP

146 conjugates secondary antibodies at room temperature for $1 \mathrm{~h}$. After three times washes, bands

147 were visualized using ECL kit. GAPDH antibody was served as a loading control.

148 Chromosome spreading and BubR1 staining 
149 Chromosome spreading was done as described previously (Chen et al., 2018). Oocytes were

150 treated with Tyrode's buffer ( $\mathrm{pH} 2.5)$ for about $30 \mathrm{~s}$ at $37^{\circ} \mathrm{C}$ to remove zona pellucidae. Then

151 oocytes were fixed in drops of speading solution (1\% PFA, 0.15\% Triton X-100, 3mM DTT in

$152 \mathrm{ddH}_{2} \mathrm{O}, \mathrm{pH}=9.2$ ) on a glass slide. After undisturbed air drying, slides were washed and blocked

153 with PBS containing 1\% BSA. Finally, samples were labeled with BubR1 antibody (Abcame,

154 1:200) and FITC-conjugated donkey anti-sheep secondary antibody (1:200). Chromosomes were 155 stained with DAPI.

156 Statistical analysis

157 Data are presented as mean \pm SEM from at least three independent replicates. Statistical analyses

158 were made with GraphPad. For statistical comparison, Student's t test was used. A value of P <

1590.05 was considered significant.

160 RESULTS

161 Cellular localization and expression pattern of OLA1 during mouse oocyte meiosis

162 Firstly, we investigated the dynamic distribution of OLA1 during mouse oocyte meiosis by 163 immunofluorescent labeling and confocal microscopy. As shown in Figure 1A, OLA1 was

164 concentrated on the whole cytoplasm at GV stage, after meiotic resume, OLA1 distributed 165 around the chromosomes and co-localized with $\alpha$-tubulin. At MI and MII stages, OLA1

166 displayed a spindle-like localization, suggesting that OLA1 may have some connections with 167 spindle assembly. To validate the OLA1 connection with spindle, MI and MII oocytes were 168 treated with nocodazole. After treatment, the spindles were depolymerized and microtubules

169 were evenly dispersed into the cytoplasm in oocytes. Meanwhile, OLA1 signal also dispersed 170 into the cytoplasm instead of around the chromosomes. Furthermore, as the oocytes were

171 thoroughly washed outof nocodazole and cultured in pre-warmed M16 medium, coupled with 
172 spindle re-assembly, OLA1 renewed its spindle-like localization, hinting that OLA1 did co-

173 localized with spindle in mouse oocyte (Figure 1C). Western blot results showed that OLA1

174 were expressed at all stages during meiotic progression (Figure 1B).

175 Knockdown of OLA1 impairs the germinal vesicle breakdown (GVBD) leading to a 176 decrease in polar body extrusion (PBE).

177 To further explore the function of OLA1 in oocyte meiosis, OLA1 specific siRNA were injected 178 into GV oocytes. As shown in Figure 2A and B, western blot analysis revealed that expression of 179 OLA1 was significantly decreased when compared with control $(\mathrm{p}<0.05)$, suggesting that 180 OLA1-RNAi achieved good knockdown efficiency for the further study of its function in oocytes. GVBD and PBE are two hallmark events in the meiotic progression. We then checked the GVBD and PBE rate after OLA1 knockdown, as shown in Figure 2C, knockdown of OLA1 significantly inhibited the GVBD progression and the average rate was $86.70 \pm 6.12 \%$ in control groups but decreased to $62.83 \pm 1.49 \%$ in OLA1-RNAi groups $(\mathrm{p}<0.05)$. After $12 \mathrm{~h}$ in culture, most of the control oocytes extruded the first polar bodies $(60.38 \pm 6.52 \%)$, while only $40.58 \pm$ $2.05 \%$ of oocytes extruded the polar body in OLA1-RNAi groups (Figure 2D, $\mathrm{p}<0.05$ ). To

187

188

190

further confirm whether the decline of PBE was due to the block of GVBD in OLA1-RNAi oocytes, we then analyzed the PBE rate in meiosis-resumed oocytes. As shown in Figure 2E, once the oocytes underwent GVBD, knockdown of OLA1 had no effect on PBE in meiosisresumed-oocytes $(69.54 \pm 3.45 \%$, control vs $64.58 \pm 2.34 \%, \mathrm{P}>0.05)$.

\section{Knockdown of OLA1 induces abnormal spindle assembly and chromosome alignment in} mouse oocyte

BRCA1 knockdown causes abnormal spindle assembly in mouse oocytes, as a direct interacting protein, OLA1 may modulate the spindle assembly. To verify this speculation, we then checked 
195 spindle morphology at metaphase stage by immunofluorescence after OLA1 knockdown. As

196 shown in Figure 3A, confocal microscopy and quantitative analysis revealed that most control

197 oocytes presented a normal barrel-shape spindle at metaphase stage, while OLA1-RNAi led to

198 abnormal multipolar and small spindles. The proportion of abnormal spindles in OLA1-RNAi

199 group was significantly higher than the control group $(30.06 \pm 0.68 \%$ vs. $11.34 \pm 1.46 \%, p<$

200 0.05; Figure 3B). Meanwhile, chromosome alignment was also disturbed in OLA1-RNAi oocyte.

201 As shown in Figure 3A, chromosomes were well-aligned at the metaphase I plate in control

202 oocytes, while OLA1-RNAi oocytes displayed irregularly scattered chromosomes (red arrow).

203 The proportion of misaligned chromosomes in OLA1-RNAi group was also significantly higher

204 than the control group $(13.19 \pm 1.59 \%$ vs. $7.92 \pm 1.57 \%, \mathrm{p}<0.05$; Figure $3 \mathrm{C})$.

205 OLA1 knockdown accelerates the anaphase onset in mouse oocytes

206 After 6 h post-GVBD in vitro culture, most of meiosis resumed-oocytes in control group have

207 reached at MI, while we found some oocytes were extruding the polar bodies in OLA1-

208 knockdown oocytes (Figure 4A). We speculate that OLA1 knockdown could result in premature

209 anaphase onset. To verify this hypothesis, we then did the cycle analysis by $\alpha$-tubulin staining

210 and confocal microscope. As shown in Figure 4B, almost all of the control oocytes reached at

211 MI, but we observed a quite number of oocytes have reached at anaphase I or telephase I in

212 OLA1-knockdown oocytes. We also recorded the proportion of oocytes that have reached at MI

213 or A/TI. Results showed that, compared with control, a lower rate of oocytes reached at MI stage

214 in OLA1-RNAi oocytes $(88.00 \pm 3.09 \%$ vs. $74.73 \pm 1.27 \%, \mathrm{p}<0.05$; Figure $4 \mathrm{C})$, while a

215 significant higher proportion of oocytes has reached A/TI stage (16.73 $\pm 2.19 \%$ vs. $2.62 \pm$

$2162.28 \%, \mathrm{p}<0.05$; Figure $4 \mathrm{C}$ ), suggesting that knockdown of OLA1 led to premature of

217 chromosome segregation and accelerated anaphase onset in mouse oocytes. 


\section{OLA1 knockdown causes premature inactivation of SAC}

219 SAC will not abrogate its activity until all the chromosomes are correctly aligned at metaphase I

220 plate, and its previous inactivation can lead to premature chromosome segregation which could

221 finally cause aneuploidy in oocytes. We have found that knockdown of OLA1 induced premature

222 of anaphase onset, we speculated OLA1 knockdown could give rise to the inactivation of SAC,

223 thus we used chromosome spreading and BubR1 (a vital component of SAC signaling) staining

224 to check SAC activity at pre-MI stage $(6.5 \mathrm{~h})$ after OLA1-RNAi. As shown in Figure 5, BubR1

225 localized to kinetochores at pre-MI stage in all control oocytes, while we could find BubR1

226 failed to be loaded onto kinetochores from kinetochores in OLA1-depletion oocytes, indicating

227 that depletion of OLA1 caused the previous inactivation of SAC at pre-MI stage.

228 OLA1 knockdown promotes the aneuploid rate

229 As we have observed misaligned chromosomes, premature anaphase trigger and SAC

230 inactivation in OLA1-knockdown oocytes, which all can induce aneuploidy in MII oocyte, so

231 we checked the aneuploid rate at MII oocyte by chromosome spreading, and the result showed

232 that the aneuploid rate was significantly increased in the OLA1-knockdown oocytes $(16.69 \pm$

$2332.51 \%$ vs. $26.24 \pm 2.51 \%$, p $<0.05$, Fig. $6 \mathrm{~B})$. These results suggest that OLA1 may participate in

234 the SAC activity, thus to contribute to the anaphase trigger and aneuploidy.

\section{DISCUSSION}

237 Spindle assembly and chromosome segregation are two indispensable events during the progress

238 of meiosis. Defective morphology of spindles and abnormal chromosome alignments could result

239 in aneuploid gametes or the failure of meiosis (Ma et al., 2014; Jiao et al., 2017; Han et al., 2015).

240 There are many factors including cellular protein function to modulate these two processes to be 
241 orderly. Currently, the function OLA1 mouse oocyte meiosis is still unknown. In this study, we

242 have discovered OLA1 is a novel participator in oocyte meiosis: the participation in GVBD,

243 spindle assembly and SAC activation.

244 GVBD and PBE are characteristic features/hallmarks of meiotic progression. When immature 245 oocytes begin to mature by exogenous and endogenous factors stimulation both in vivo and in 246 vitro, GVBD occurs and then oocyte resumes meiosis which could finally bring out the polar 247 body extrusion (Sánchez et al., 2012). Defects in GVBD can result in the block of meiosis and 248 oocyte will lose their ability for further development. To detect the function of OLA1, we first 249 examined GVBD and PBE rates after OLA1 knockdown. We found that OLA1 knockdown 250 significantly inhibited GVBD and a decline in PBE was also found in the loss of OLA1.

251 However, once the oocyte underwent GVBD then it could directly extrude the polar body even in 252 the loss of OLA1. And the decline of PBE in OLA1 knockdown oocytes was only due to the 253 block of GVBD. Thus, we conclude that OLA1 is critical for the germinal vesicle breakdown but 254 not polar body extrusion.

255 Spindle assembly is indispensable for meiotic progression, it supports the segregation of 256 chromosomes in oocytes. Abnormal spindle assembly could result in PBE failure or production 257 of aneuploid gametes (Schuh et al., 2007; Clift et al., 2015; Bennabi et al., 2016). During the time of 258 spindle assembly, chromosomes show unstable connections with microtubules until hours post 259 GVBD when a tight link between kinetochores and microtubules is established. To avoid the 260 segregation of chromosomes with incorrect connection with microtubules, SAC persist at 261 kinetochore (SAC activation) to prevent the premature of chromosome segregation (Vogt et al., 262 2008; Touati et al., 2016; Sanders et al., 2018). Once all chromosomes align correctly at the 263 metaphase I plate, SAC proteins will dissociated from kinetochores (SAC inactivation) then 
264 activates the APC/C activity to accelerate SECURIN degradation and anaphase I onset (Marston 265 et al., 2017 ). Defects in SAC function can result in incorrect attachments of chromosome with 266 microtubules, and leads to missegregation (Miao et al., 2017; Lu et al., 2017; Li et al., 2009). Study

267 has shown that knockdown of BRCA1 causes abnormal spindles in mouse oocytes (Xiong et al., 268 2008). As an interactive protein in mitosis, we speculated OLA1 may also function in spindle 269 assembly in oocyte. Results showed that multipolar and small spindles occurred in OLA1270 knockdown oocytes, indicating that OLA1 also participate in spindle assembly in meiosis.

271 What's more, we speculate that OLA1 could bind to BRCA1 in oocyte exerting its regulatory 272 function in meiotic spindle assembly, but still needs further study to verify that. In addition, a 273 higher proportion of oocytes have reached A/TI stage after $8 \mathrm{~h}$ in culture, indicating the 274 premature of anaphase onset in OLA1-knockdown oocytes. Further study identified the 275 inactivation of SAC at pre-MI stage in RNAi oocytes, thus knockdown of OLA1 results in the 276 dysfunction of SAC leading to the premature chromosome segregation. Importantly, knockdown 277 of BRCA1 in mouse oocyte also leads to the inactivation of SAC (Xiong et al., 2008). Taken all 278 these together, it seems that OLA1 may function in meiosis in an OLA1-BRCA1 mediated 279 pathway. In addition, OLA1 knockdown induced spindle defects did not lead to metaphase I 280 arrest, we speculate this might be the bypass of SAC supervision.

\section{CONCLUSION}

282 In brief, our study highlights that OLA1 exhibits significant function in meiotic progression, 283 especially in GVBD regulation. OLA1 also functions in spindle assembly and SAC activation.

\section{REFERENCES}

285 Bennabi I, Terret ME, Verlhac MH. 2016. Meiotic spindle assembly and chromosome 286 segregation in oocytes. J. Cell Biol. 215(5):611-619 DOI 10.1083/jcb.201607062. 
Chen F, Jiao XF, Zhang JY, Wu D, Ding ZM, Wang YS, Miao YL, Huo, LJ. 2018. Nucleoporin 35 is a novel microtubule associated protein functioning in oocyte meiotic spindle architecture. Exp. Cell Res. 371 (2):435-443 DOI 10.1016/j.yexcr.2018.09.004.

Chen H, Song R, Wang G, Ding Z, Yang C, Zhang J, Zeng Z, Rubio V, Wang L, Zu N, Weiskoff AM, Minze LJ, Jeyabal PV, Mansour OC, Bai L, Merrick WC, Zheng S, Shi ZZ. 2015. OLA1 regulates protein synthesis and integrated stress response by inhibiting eIF2 ternary complex formation. J. Scientific Reports. 5(10):13241DOI 10.1038/srep13241.

Clift D, Schuh M. 2015. A three-step MTOC fragmentation mechanism facilitates bipolar spindle assembly in mouse oocytes. Nat. Commun. 6:7217 DOI 10.1038/ncomms8217.

Ding Z, Liu Y, Rubio V, He J, Minze LJ, Shi ZZ. 2016. OLA1, a translational regulator of p21, maintains optimal cell proliferation necessary for developmental progression. Molecular $\boldsymbol{\&}$ Cellular Biology. 36(20):2568-2582 DOI 10.1038/srep13241

Clift D, Schuh M. 2015. A three-step MTOC fragmentation mechanism facilitates bipolar spindle assembly in mouse oocytes. Nat. Commun. 6:7217 DOI 10.1038/ncomms8217.

Han L, Ge J, Zhang, L, Ma R, Hou X, Li B, Moley, K, Wang Q. 2015. Sirt6 depletion causes spindle defects and chromosome misalignment during meiosis of mouse oocyte. Sci. Rep. 5:15366 DOI 10.1038/srep15366.

Jiao XF, Huang CJ, Wu D, Zhang JY, Long YT, Chen F, Li X, Huo LJ. 2017. Abce1 orchestrates M-phase entry and cytoskeleton architecture in mouse oocyte. Oncotarget $\mathbf{8 ( 2 4 ) :}$ 39012-39020 DOI10.18632/oncotarget.16546.

Koller-Eichhorn R, Marquardt T, Gail R, Wittinghofer A, Kostrewa D, Kutay U, Kambach C. 2007. Human OLA1 defines an ATPase subfamily in the Obg family of GTP-binding proteins. J. Biol. Chem.282(27):19928-19937 DOI 10.1074/jbc.M700541200. 
310 Li M, Li S, Yuan J, Wang ZB, Sun SC, Schatten H, Sun QY. 2009. Bub3 is a spindle

311 assembly checkpoint protein regulating chromosome segregation during mouse oocyte

312 meiosis. PLoS One 4 (11):e7701 DOI 10.1371/journal.pone.0007701.

313 Lu Y, Dai X, Zhang M, Miao Y, Zhou C, Cui Z, Xiong B. 2017. Cohesin acetyltransferase

314 Esco2 regulates SAC and kinetochore functions via maintaining H4K16 acetylation during

315 mouse oocyte meiosis. Nucleic Acids Res. 45(16):9388-9397 DOI 10.1093/nar/gkx563.

316 Ma R, Hou X, Zhang L, Sun SC, Schedl T, Moley, K, Wang Q. 2014. Rab5a is required for

317 spindle length control and kinetochore-microtubule attachment during meiosis in oocytes.

318 FASEB J. 28(9):4026-4035 DOI 10.1096/fj.14-250886.

319 Marston AL, Wassmann K. 2017. Multiple duties for spindle assembly checkpoint kinases in 320 meiosis. Front. Cell Dev. Biol. 5:109 DOI 10.3389/fcell.2017.00109.

Matsuzawa A, Kanno S, Nakayama M, Mochiduki H, Wei L, Shimaoka T, Furukawa Y, protein OLA1 functions in centrosome regulation. Mol. Cell 53 (1):101-114 DOI

Sánchez F, Smitz J. 2012. Molecular control of oogenesis. Biochim. Biophys. Acta. 1822 10.1016/j.molcel.2013.10.028.

Miao Y, Zhou C, Cui Z, Dai X, Zhang M, Lu Y. Xiong B. 2017. Smc1 $\beta$ is required for activation of SAC during mouse oocyte meiosis. Cell Cycle 16(6):536-544 DOI $10.1080 / 15384101.2017 .1282583$ (12):1896-1912 DOI 10.1016/j.bbadis.2012.05.013.

Sanders JR, Jones KT. 2018. Regulation of the meiotic divisions of mammalian oocytes and eggs. Biochem. Soc. Trans. 46(4):797-806 DOI 10.1042/BST20170493. 
332 Schuh M, Ellenberg J. 2007. Self-organization of MTOCs replaces centrosome function during

333 acentrosomal spindle assembly in live mouse oocytes. Cell 130(3):484-498 DOI

$334 \quad$ 10.1016/j.cell.2007.06.025.

335 Touati SA, Wassmann K. 2016. How oocytes try to get it right: spindle checkpoint control in 336 meiosis. Chromosoma 125(2):321-335 DOI 10.1007/s00412-015-0536-7.

337 Verlhac MH, Terret ME. 2016. Oocyte Maturation and Development. F1000 Faculty Rev:309

338 DOI 10.12688/f1000research.7892.1.

339 Vogt E, Kirsch-Volders M, Parry J, Eichenlaub-Ritter U. 2008. Spindle formation,

340 chromosome segregation and the spindle checkpoint in mammalian oocytes and

341 susceptibility to meiotic error. Mutat. Res.651(1-2):14-29 DOI

$342 \quad$ 10.1016/j.mrgentox.2007.10.015.

343 Webster A, Schuh M. 2017. Mechanisms of Aneuploidy in Human Eggs. Trends Cell Biol. $344 \quad$ 27(1):55-68 DOI 10.1016/j.tcb.2016.09.002.

345 Xiong B, Li S, Ai JS, Yin S, Ouyang YC, Sun SC, Chen DY, Sun QY. 2008. BRCA1 is

346 required for meiotic spindle assembly and spindle assembly checkpoint activation in mouse

347 oocytes. Biol. Reprod. 79 (4):718-726 DOI 10.1095/biolreprod.108.069641.

348 Yoshino Y, Qi H, Fujita H, Shirota M, Abe S, Komiyama Y, Shindo K, Nakayama M,

349 Matsuzawa A, Kobayashi, A, Ogoh, H, Watanabe T, Ishioka C, Chiba N. 2018. BRCA1-

350 interacting protein OLA1 requires interaction with BARD1 to regulate centrosome number.

351 Mol. Cancer Res.16(10):1499-1511 DOI 10.1158/1541-7786.MCR-18-0269.

352 Zhang J, Rubio V, Lieberman MW, Shi, ZZ. 2009. OLA1, an Obg-like ATPase, suppresses

353 antioxidant response via nontranscriptional mechanisms. Proc. Natl. Acad. Sci. USA

354 106(36):15356-15361 DOI 10.1073/pnas.0907213106. 


\section{Figure Legends}

356 Figure 1 OLA1 localization and expression during mouse oocyte meiosis. (A) Subcellular

357 localization pattern of OLA1 in mouse oocyte meiosis was explored by immunofluorescent

358 analysis. Samples were harvested at GV, GVBD, MI, and MII stage and then immunolabeled.

359 OLA1, red; $\alpha$-tubulin, green and Chromosome, blue. Scale bar, $10 \mu \mathrm{m}$. (B) Subcelluar expression

360 pattern of OLA1 in mouse oocyte meiosis was investigated by western blot analysis. Samples at

361 indicated stages were collected. (C) Confocal images of OLA1 signal in MI/MII oocytes after

362 treatment with nocodazole and recovery. OLA1, red; $\alpha$-tubulin, green and Chromosome, blue.

363 Scale bar, $10 \mu \mathrm{m}$.

364 Figure 2 Knockdown of OLA1 impairs GVBD leading to a decrease in PBE. Fully grown

365 oocytes injected with OLA1 siRNA or control siRNA were arrested in M2 medium with IBMX

366 for 24 hours, then cultured in vitro for the continuous experiments. (A, B) Knockdown efficiency

367 of OLA1 after OLA1 siRNA was verified by western blot; (C, D) Oocytes after microinjection

368 were released into M16 medium for further culture. The GVBD rate in control group and OLA1

369 knockdown group were recorded at $2 \mathrm{~h}$; The PBE rate in control group and OLA1 knockdown

370 group were recorded at $12 \mathrm{~h} ;{ }^{*} \mathrm{p}<0.05$. (E) PBE rate was characterized in meiosis resumed

371 oocytes in control and RNAi groups. 162 control oocytes and 182 OLA1-RNAi oocytes were

372 calculated.

373 Figure 3 OLA1 knockdown leads to defects of spindle assembly and chromosome

374 alignment. (A) Spindle morphology in control and OLA1-depletion oocytes after $6 \mathrm{~h}$ post-

375 GVBD culture. $\alpha$-tubulin, green; Chromosome, blue. Scale bar, $10 \mu \mathrm{m}$; (B) Quantification of

376 aberrant spindles rate in control and OLA1-RNAi oocytes; (C) Quantification of misaligned 
377 chromosomes rate in control and OLA1-RNAi oocytes. 88 control oocytes and 90 OLA1-RNAi

378 oocytes were calculated in abnormal spindles and misaligned chromosomes. ${ }^{*} \mathrm{p}<0.05$.

379 Figure 4 OLA1 knockdown accelerates the anaphase onset in mouse oocytes. (A) Images

380 exhibited different status of the first polar extraction in control and OLA1-depletion groups; (B)

381 The spindle and chromosome morphologies in control and OLA1-knockdown oocytes after $6 \mathrm{~h}$

382 post-GVBD culture. $\alpha$-tubulin, green; Chromosome, blue. Scale bar, $10 \mu \mathrm{m}$; (C) Cell cycle

383 analysis in control oocytes $(n=97)$ and OLA1-RNAi oocytes $(n=92)$ after 6 h post-GVBD

384 culture. $* \mathrm{p}<0.05$

385 Figure 5 OLA1 knockdown causes precocious inactivation of SAC. The status of BubR1 at 386 pre-MI stage from control and OLA1-knockdown oocytes. After knockdown, GV oocytes in 387 control and OLA1-knockdown groups were released and cultured in pre-warmed M16 medium 388 for $6.5 \mathrm{~h}$, normally corresponding to Pre-MI stage. Then oocytes were collected for chromosome 389 spreading and stained with BubR1. BubR1, green; Chromosome, Blue. Scale bar, $20 \mu \mathrm{m}$.

390 Figure 6 OLA1 knockdown increases the aneuploid rates. (A) Confocal images of

391 chromosome spread of MII oocytes. Control oocytes with a normal haploid complement of 20

392 chromosomes, OLA1 RNAi oocytes with 16 and 19 chromosomes. Chromosome, Blue. Scale

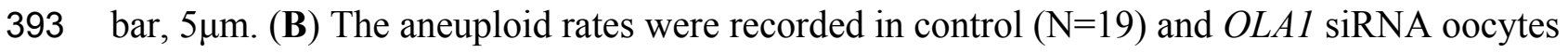
$394(\mathrm{~N}=22) .{ }^{*} \mathrm{p}<0.05$. 
Figure 1

Cellular localization and expression pattern of OLA1 in mouse oocyte meiosis

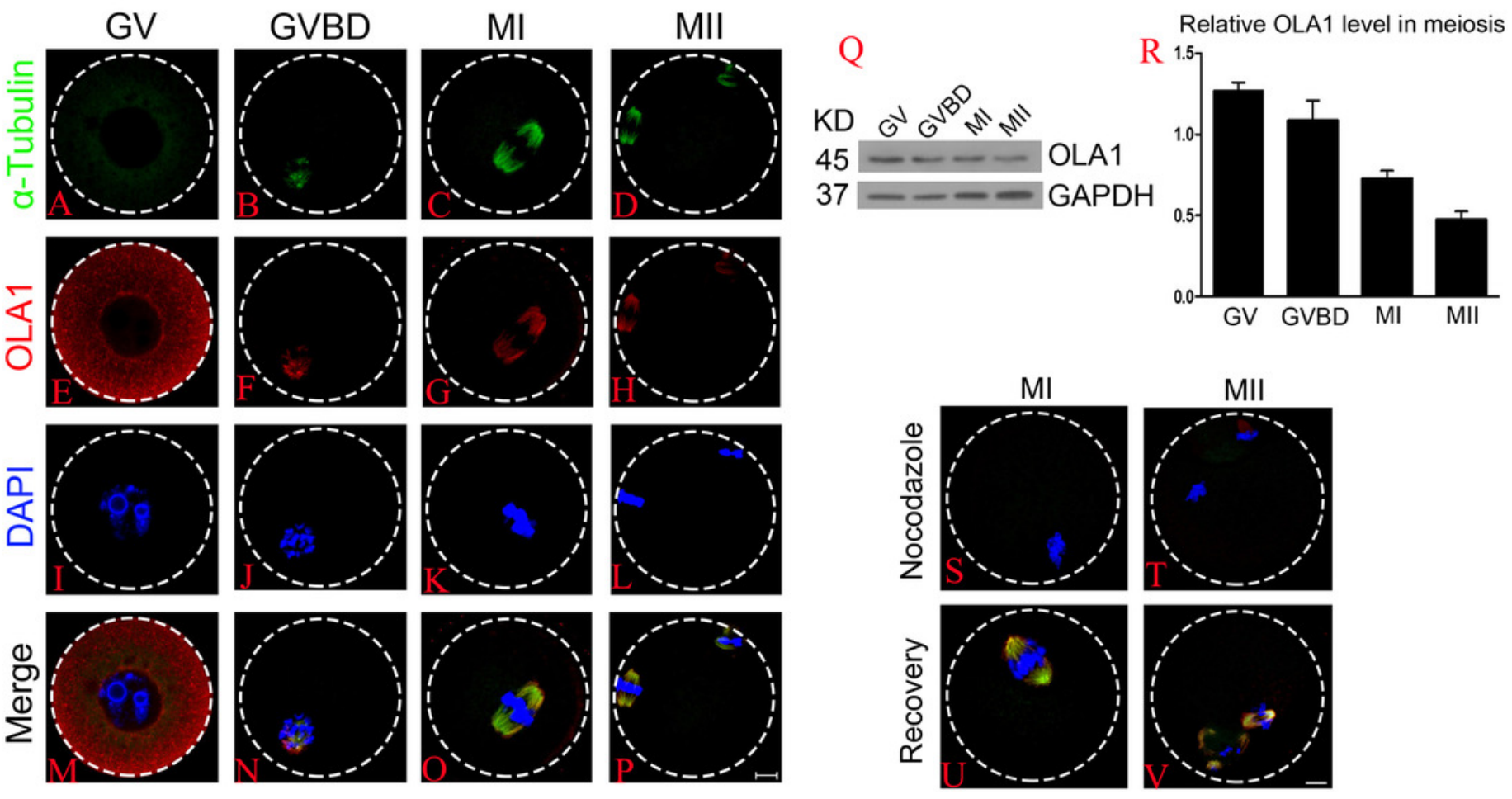


Figure 2

Knockdown of OLA1 impairs GVBD leading to a decrease in PBE

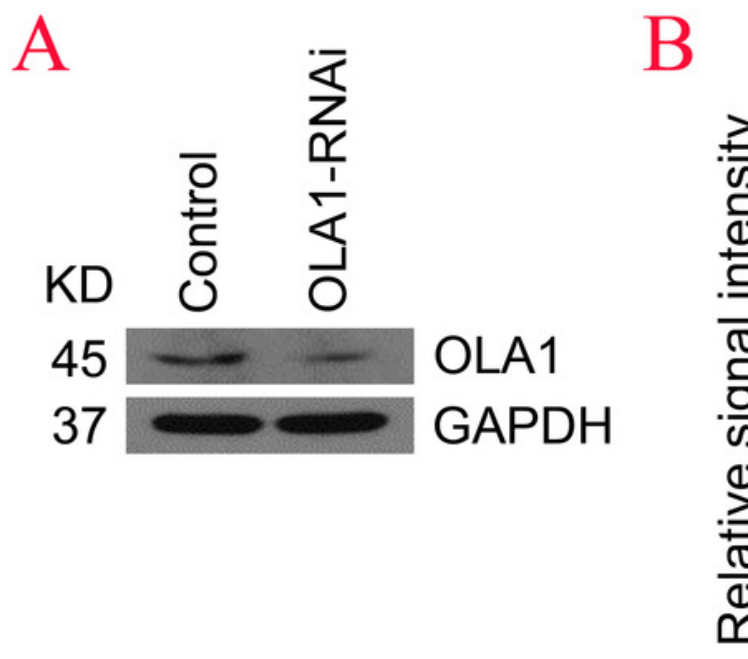

$\mathrm{D}$

$12 \mathrm{~h}$ in culture

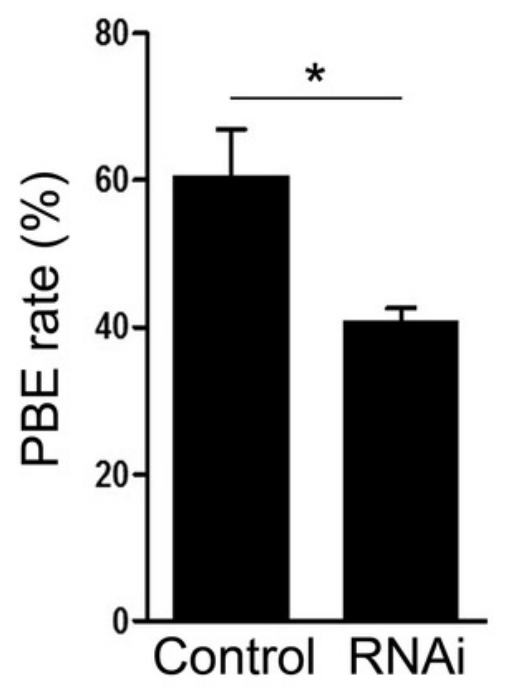

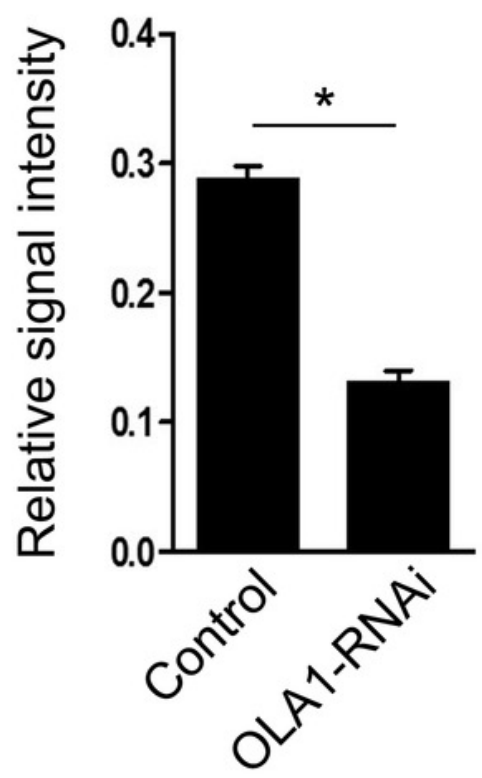

C $2 \mathrm{~h}$ in culture

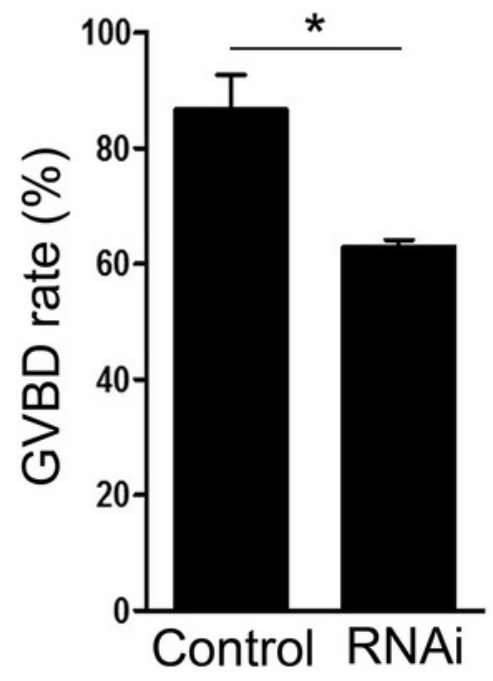

E GVBD/PBE Rate

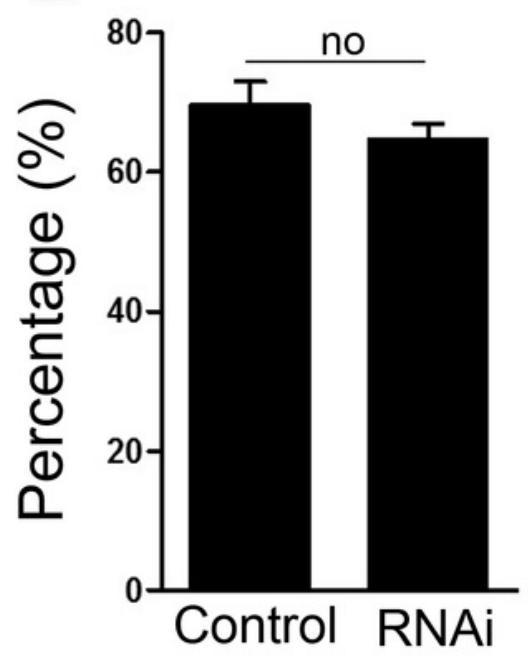


Figure 3

OLA1 is required for spindle assembly and chromosome alignment in mouse oocytes
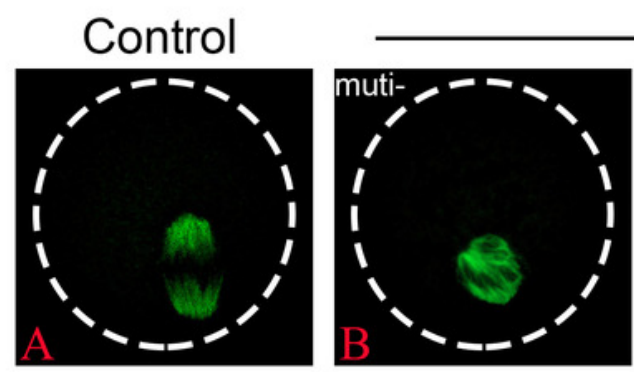

OLA1-RNAi-
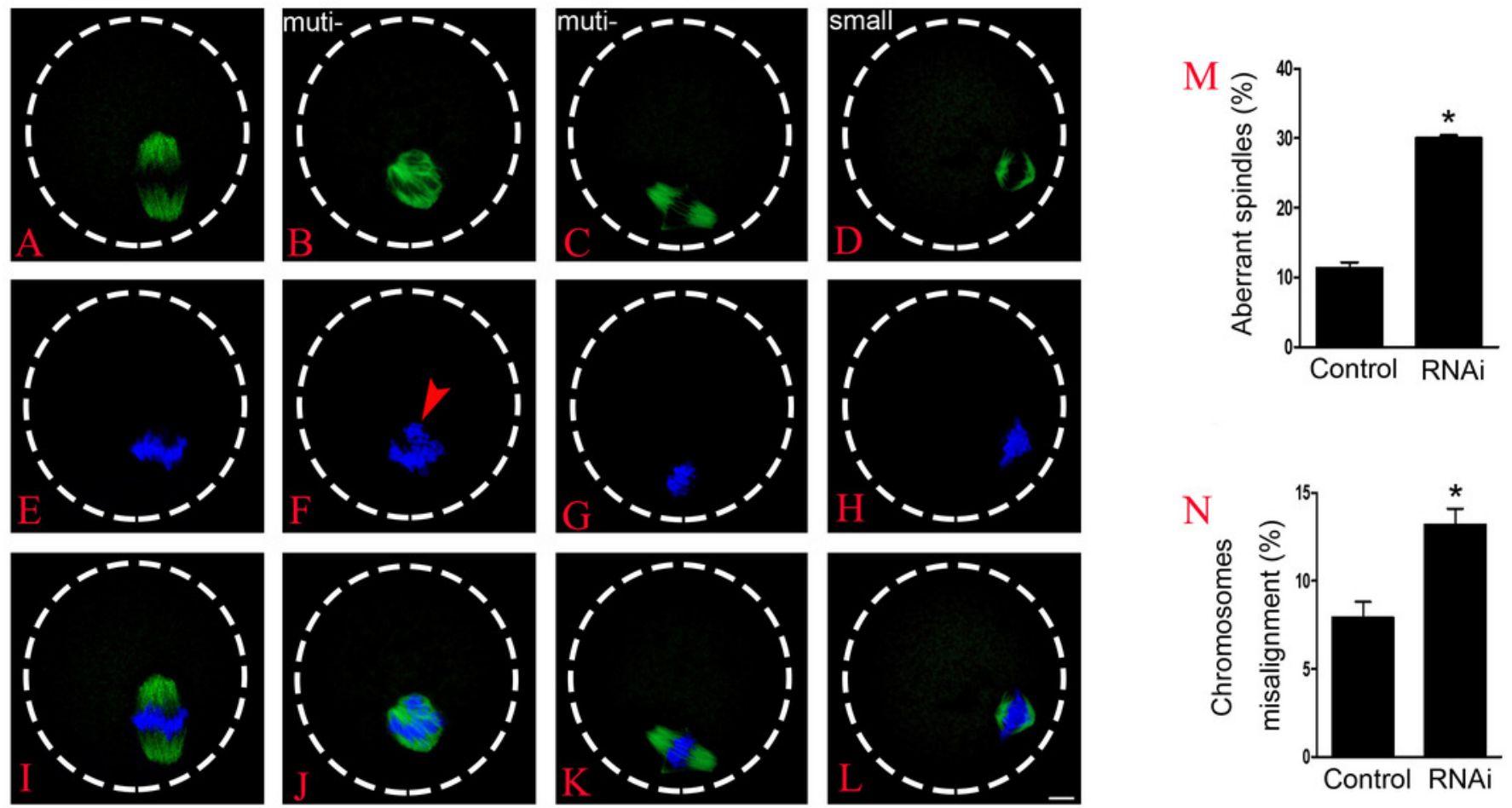
Figure 4

OLA1 knockdown accelerates the anaphase onset in mouse oocytes
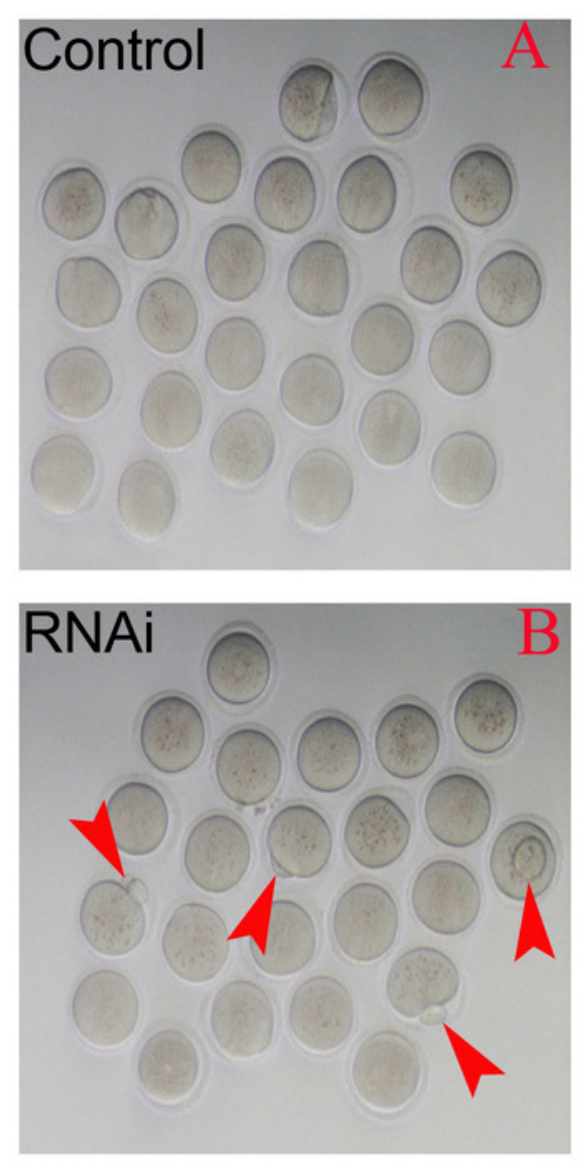
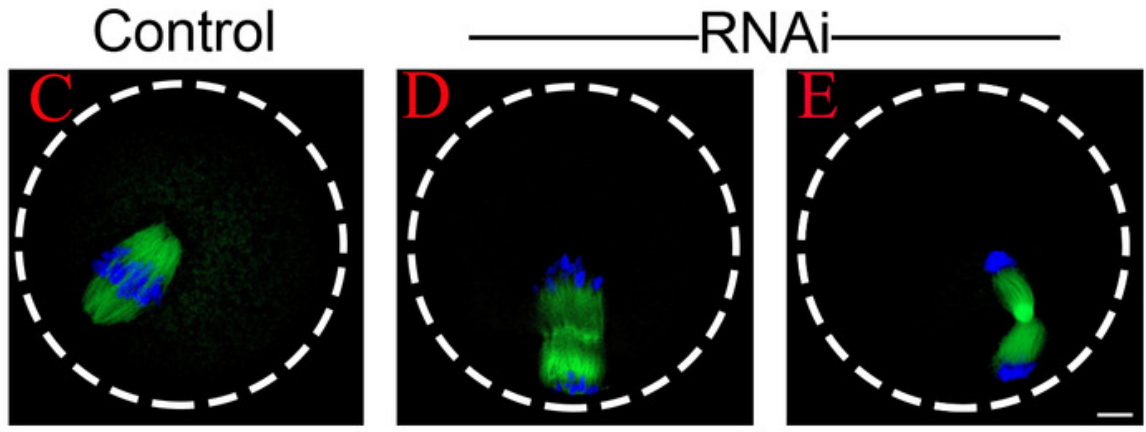

$\mathrm{F}$

Cell cycle for meiosis-resumed oocytes after $6 \mathrm{~h}$ in culture

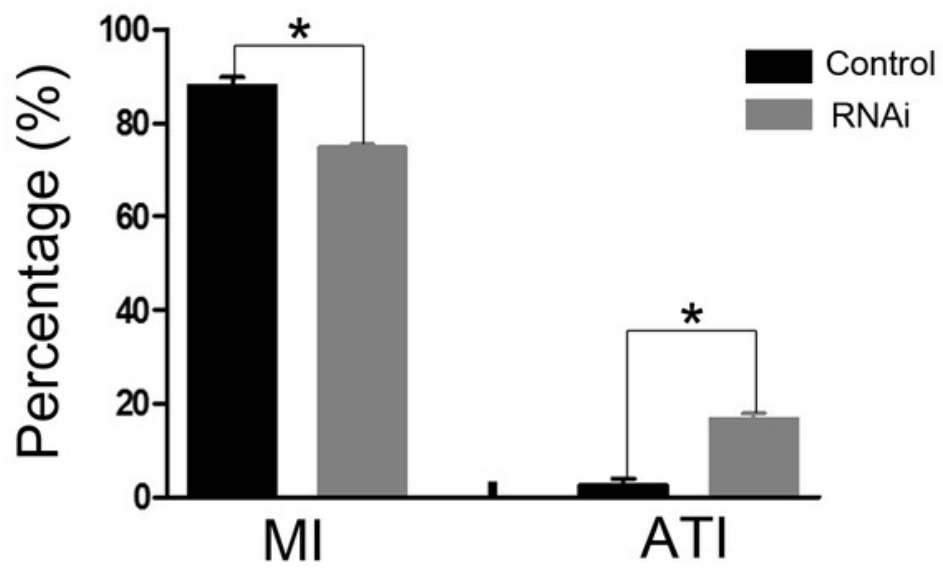




\section{Figure 5}

OLA1 knockdown causes inactivation of spindle assembly checkpoint

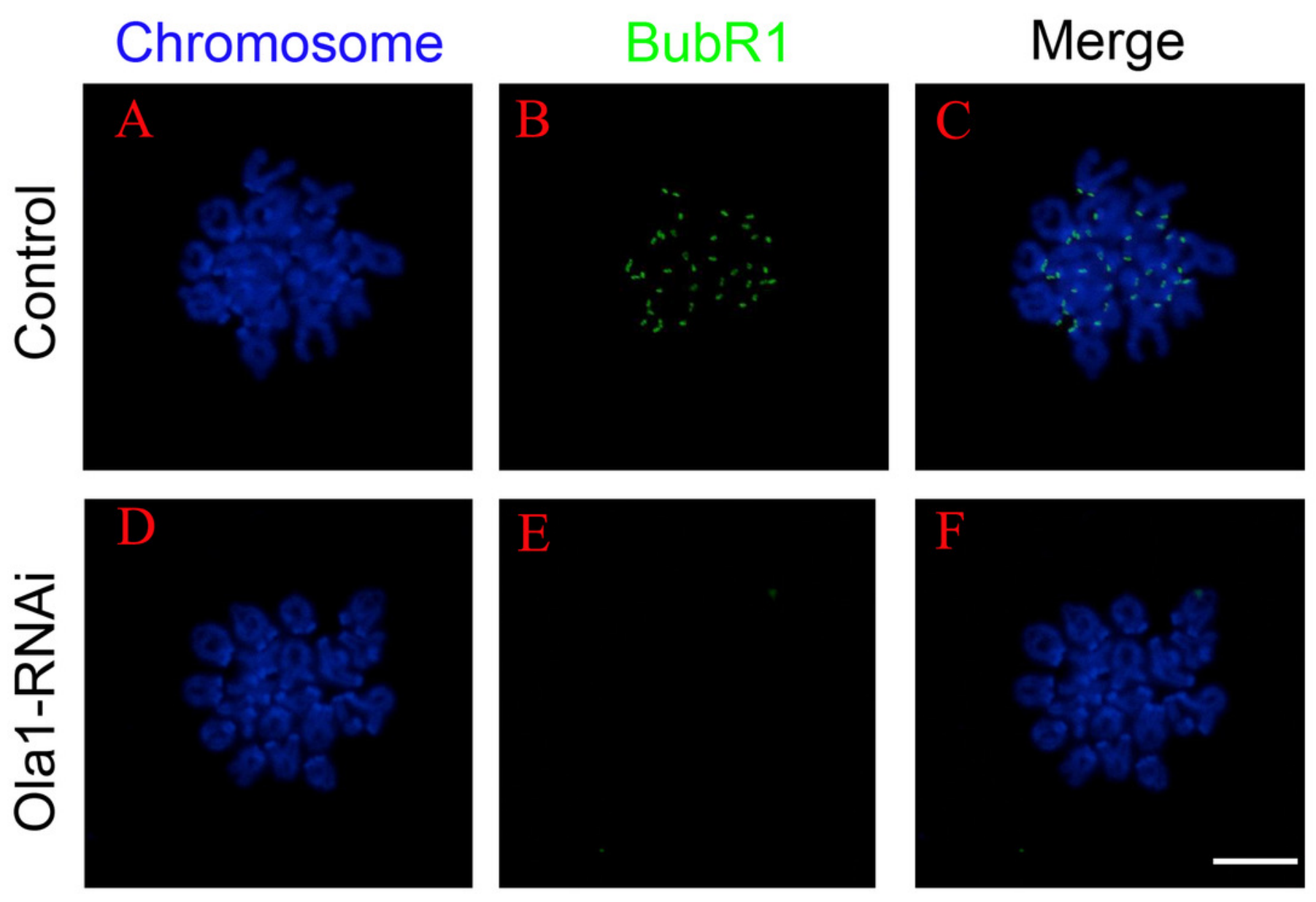


Figure 6

OLA1 knockdown promotes the aneuploid rate

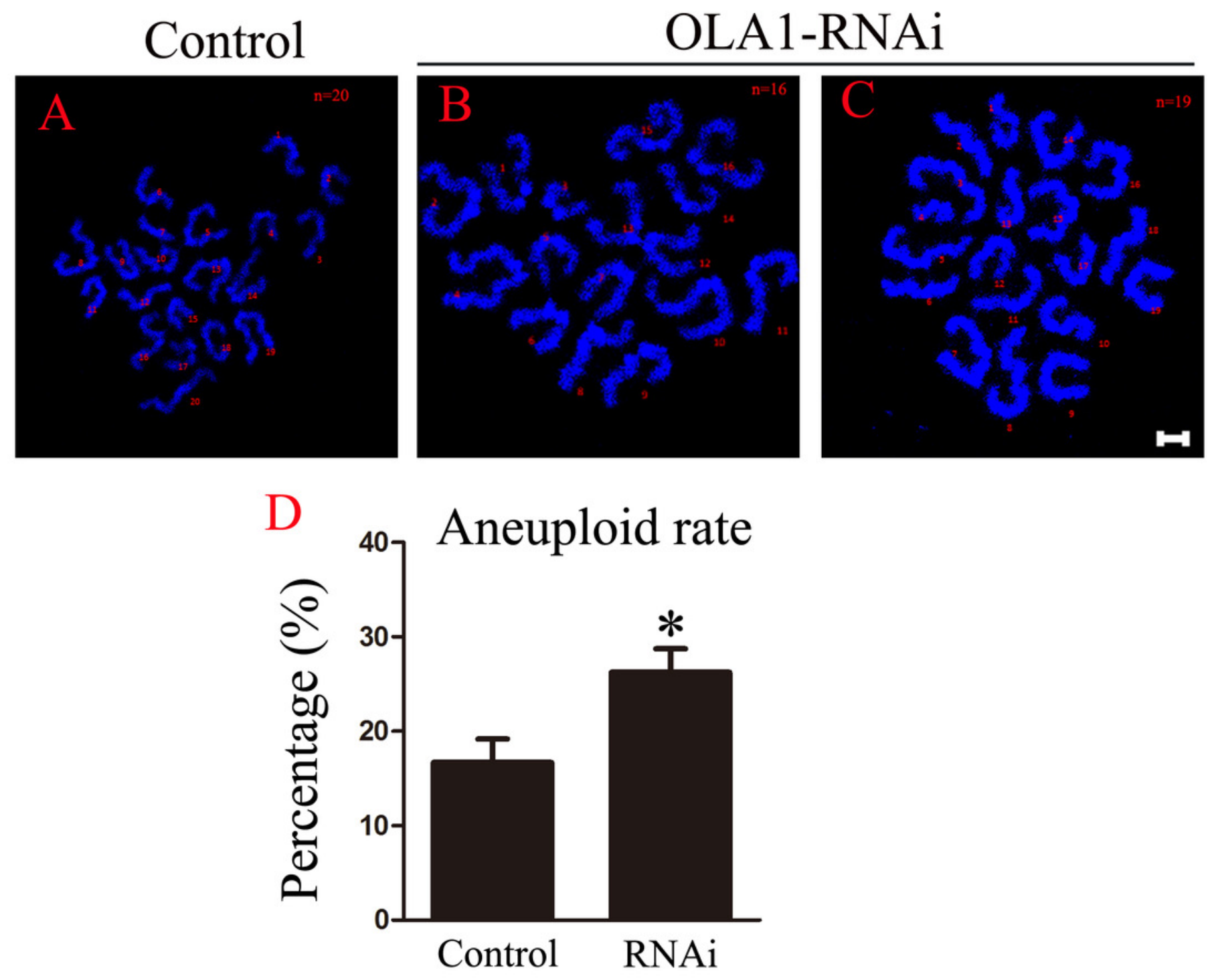

